\title{
Evaluation of System Performance of Ultra-Wide Band Jointing in MC Signaling in Correlated Environments
}

\author{
Joy Iong-Zong Chen ${ }^{1 *}$, Kai-Chih Chuang ${ }^{1}$, Lu-Tsou Yeh ${ }^{2}$ \\ ${ }^{1}$ Department of Communication Engineering, Dayeh University, Chunghua, Taiwan \\ ${ }^{2}$ Department of Electrical Engineering, Asia-Pacific Institute of Creativity, Miaoli, Taiwan \\ Email: ${ }^{j}$ jchen@mail.dyu.edu.tw
}

Received February 5, 2013; revised March 6, 2013; accepted March 13, 2013

Copyright (C) 2013 Joy Iong-Zong Chen et al. This is an open access article distributed under the Creative Commons Attribution License, which permits unrestricted use, distribution, and reproduction in any medium, provided the original work is properly cited.

\begin{abstract}
The evaluation of System Performance of UWB (ultra-wide band) jointing in MC (multi-carrier) signaling in correlated environments is presented in the report. The correlated Nakagami- $m$ statistical distribution for the multipath fading model is assumed in this scenario. In fact to establish the model for analyzing in this article is using MC-CDMA (multi-carrier code-division multiple-access) system characterization combined with a UWB scheme. The average BER (bit error rate) is calculated and compared to a special case of previously published results. Studied results from this paper can be implied to approve the system performance for a UWB system combined with a MC-CDMA wireless communication system. It is worth noting that the Nakagami- $m$ distributed fading parameter significantly dominates UWB system performance when it cooperates with MC signaling under a fading environment. Finally, it is worthy of noting that when the SNR (signal-to-noise ratio) at system's receiver reaches a preset high threshold value, the parameter of power decay ratio effect could be not included.
\end{abstract}

Keywords: MC Signaling; Multipath; Correlated Nakagami- $m$ Distribution; UWB (Ultra-Wide Band)

\section{Introduction}

The UWB (ultra-wide band) system is explained as a system which the impulse wireless radio system transmits data without a sinusoidal modulating signal, it is instead of sub-nanosecond pulses, recently raised growing interest. The UWB system, which has joint bandwidth in excess $1 \mathrm{GHz}$ with very low PSDs (power spectral densities) but without inducing significant extra interference to incumbent subscribers, has emerged as a solution for the TG3a (IEEE 802.15a) standard to provide low complexity, low power consumption, low cost and high data rate among WPAN (Wireless Personal Area Networking) devices. The modulating sub-nanosecond pulses obtain a $10 \mathrm{~dB}$ bandwidth benefit which exceeds $500 \mathrm{MHz}$ or on the order of one to several Gigahertz and is traditionally $20 \%$ of their center frequency. It is known that UWB signals have large bandwidth when appropriately modulated with spreading spectrum techniques and provide low error intercept and detection probability as well as robustness to jamming. UWB signals occupy large bandwidth and account mainly for both the drawback and

${ }^{*}$ Corresponding author. advantages of UWB radio systems. For the purpose of minimizing interference to these systems, UWB system operation must strictly follow certain necessary limitations, e.g., transmission range, power control implementation and achievable data rate. In order to break through these limitations, UWB systems could be developed to coexist with wideband and narrowband systems already allocated for a dedicated frequency spectrum, such as multi-carrier modulated schemes, SS-CDMA (spreadspectrum code-division multiple-access), DS-CDMA (direct-sequence CDMA) or MB-OFDM (multi-band orthogonal frequency division multiplexing) systems. UWB channels are characterized as frequency selective while the waveform of UWB system propagates over a fading channel, because of their extremely high bandwidth, as reported in [1]. To achieve higher data rates with lower power consumption and support multiple users, the DS-SS (direct-sequence spread-spectrum) has been promoted to reach this previously mentioned target. Several researchers have addressed in this issue. Recently, in [2] the author introduced a new UWB approach with DS-UWB signaling and evaluated the system performance. The performance of a DS-UWB system over 
multipath fading environments was evaluated in [3] at about the same duration. MC-CDMA system performance was investigated in the presence of narrowband interference for future UWB communication in [4]. In [5] the authors outlined some attractive features of TH-SS (time hopping spread-spectrum) multiple-access system and estimated the multiple-access capability for both ana$\log$ and digital data modulation schemes under ideal channels. In [6] a frequency selective Nakagami fading channel was considered to analyze inter-symbol/frame interference for pulsed DS (direct sequence) and hybrid DS-TH (DS-time hopping) UWB communications. In [7] the channel models for UWB were overviewed for UWB propagation channels. Deterministic and stochastic models were also described for UWB channels and with key parameters identified for the measurement description. The researchers showed results from the developed equalization in [8], claiming that a linear equalization scheme with widely linear processing is well suited for DS-UWB system lower data rate modes, whereas nonlinear equalization is preferable for high-data rate modes. A novel approach for reducing the power consumption and complexity of a MB-OFDM (multiband orthogonal frequency-division multiplexing) UWB system involved applying ideas from pulsed UWB systems was described in [9]. A decentralized cooperative beam-forming scheme was proposed in [10] where the impulse-based UWB is designed to link on cooperative wireless ad hoc networks. The system performance of PCSS-UWB (parallel combinatory spread spectrum) with multiple users was evaluated considering the AWGN channel in [11], where the authors also proposed MUD (multiple user detection) in the system with $2 \mathrm{~dB}$ outperforming the system without MUD. In [12] for suppressing the mutual interference between ultra-wide band-width wireless and coexisting narrowband services, DS and THS designs were proposed for impulse radio ultra-wide bandwidth systems.

The aforementioned research motivated this paper's contribution in analyzing MC (multi-carrier) techniques combined with the UWB system performance over the correlated-Nakagami- $m$ fading channel. The correlation phenomenon caused by the distance between subchannels is not sufficient, a fact validated in [13]. There are several closed-form BER (bit-error rate) derived formulas. Because most UWB systems are deployed in short distance indoor environments, it is believed that the correlation effect between the multipath branches could dominate the performance. On the other hand, the correlation fact is not negligible when UWB scheme performance evaluation is conducted. This paper is organized as follows: the system model is presented in Section 2. Section 3 analyzes UWB system with MC-CDMA techniques performance. The numerical results and dis- cussion are illustrated in Section 4. A simple conclusion is drawn in Section 5.

\section{System Models}

It is known that the MC (multi-carrier) system can be incorporated in the UWB system, e.g. the MC-CDMA system overlay for the UWB communication system [4]. This situation assumes that the bandwidth of each MC system subcarrier is $W_{\text {sub }}=W_{u} / N$, where $W_{u}$ and $N$ denote the UWB system bandwidth and the number of subcarriers, respectively. We evaluate the system performance for a typical UWB system based on TH-PPM (time-hopping pulse position modulation) scheme, which is the most popular, suitable modulation technique for the UWB system [1], combined with the MC-CDMA system and the working environment for the propagated channel. The binary source data sequence, $\alpha_{\left\lfloor i / N_{s}\right\rfloor}^{(k)}$, where $\left\lfloor i / N_{s}\right\rfloor$ indicates the integer part of $i / N_{s}$, of the $k$-th user is first spread by a set of random time-hopping sequences, $c_{i}^{(k)}$, with processing gain $P G=T_{b} / T_{h}$, where $T_{b}$ and $T_{h}$ stand for the bit duration and the hopping time, respectively.

Based on TH-PPM scheme utilization for the UWB system, the BER of the coherent BPSK (binary phase shift keying) modulation with AWGN (additive white Gaussian noise) channel is calculated in [1], and rewritten as

$$
B E R=\frac{1}{2} \operatorname{erfc}\left(\sqrt{\frac{E_{b}}{N_{0}} \frac{1-R(t)}{2}}\right)
$$

where $R(t)$, in which $t$ represents the time shift of the comparison between the pulse in a data bit " 1 " to a data bit " 0 ", is defined as the auto-correlation function of the received pulse signal and $E_{b} / N_{0}$ represents the SNR (signal-to-noise ratio) of the frame. The BER system for evaluating the communication system with BPSK modulation can be expressed as

$$
B E R=\frac{1}{2} \operatorname{erfc}\left(\sqrt{\frac{E_{b}}{N_{0}} \frac{T_{i}}{T_{i}+T_{g}}}\right)
$$

where $T_{g}$ is the guard interval time, $T_{i}$ indicates the bit symbol time without guard interval time 1 (1 Since PPM (pulse position modulation) is adopted for UWB system, guard interval exists between each pulse position is necessary). Consider a channel that has frequencyselective fading, the channel impulse response is then modeled as a linear filter and can be written as

$$
h(t)=\sum_{\ell=0}^{L-1} h_{\ell} \delta\left(t-\tau_{\ell}\right)
$$

where $h_{\ell}=\alpha_{\ell} \exp \left(j \phi_{\ell}\right), \ell=0, \cdots, L-1$ are mutually un- 
correlated, $\delta(\cdot)$ denotes the Dirac delta function, $\tau_{\ell}$ is the time delay of the 1-th diversity path, the phase $\phi_{\ell}, \ell=0, \cdots, L-1$, are uniformly distributed over $(0,2 \pi]$, and the amplitude $\alpha_{\ell}=\left|h_{\ell}\right|$ is assumed characterized as Nagakami- $m$ distributed, which is denoted by $\alpha_{\ell} \sim M\left(\alpha_{\ell} ; m_{\ell}, \Omega_{\ell}\right)$ [14], which is restated as

$$
\begin{aligned}
& M\left(\alpha_{\ell} ; m_{\ell}, \Omega_{\ell}\right) \\
& =\frac{2}{\Gamma\left(m_{\ell}\right)}\left(\frac{m_{\ell}}{\Omega_{\ell}}\right)^{m_{\ell}} \alpha_{\ell}^{2 m_{\ell}-1} \exp \left(-\frac{m_{\ell}}{\Omega_{\ell}} \alpha_{\ell}^{2}\right), \\
& \ell=0, \cdots, L-1
\end{aligned}
$$

where $\Gamma(\cdot)$ is the Gamma function, $\Omega_{\ell}=E\left[\left|h_{\ell}\right|^{2}\right]$ denotes the average power of the received signal and $m_{\ell}, \ell=0, \cdots, L-1$ are the fading parameters, with $m_{\ell} \geq 1 / 2$ representing the fading severity. The smaller the $m_{\ell}$ values are, the greater the fading in the channel environment. Assuming that the fading parameters of all taps are identical, i.e., $m_{i}=m_{j}$ for

$i \neq j, i, j=0, \cdots, L-1$ and exponential MIP (multipath intensity profile), $\Omega_{\ell}=\Omega_{0} \mathrm{e}^{-\ell \delta}$, where $\Omega_{0}$ is the average power of the first channel path, $\delta \geq 0$ indicates the average power decay rate, however, $\delta=0$ corresponds to the constant MIP condition. To claim that the pulsed-UWB channel is modeled as correlated Nakagami-m fading in this report is necessary. Generally the relevant UWB techniques are applied in a short distance and the standard channel model with IEEE 802.15.3a is always the one model suitable for the UWB system. It is reasonable to adopt the correlated-Nakagami- $m$ distributed system as the channel model in the indoor communications case [7]. The channel could suffer from the correlation between the diversity paths due to not having enough subchannel separation. The N-point $(N \geq L)$ DFT (discrete Fourier transform) of the channel impulse response at nth frequency bin is defined as

$$
H_{n}=\frac{1}{\sqrt{N}} \sum_{\ell=0}^{L-1} h_{\ell} \exp \left(-j \frac{2 \pi n \ell}{N}\right), 0 \leq n \leq N-1 .
$$

The approximate $\beta_{n}=\left|H_{n}\right|$ expression is adopted to analyze the system performance showing that the fading intensity follows the Nagakami- $m$ distribution with fading parameter $m_{f}$, and mean power $\Omega_{f}$, i.e., $\beta_{n} \sim M\left(\beta_{n} ; m_{f}, \Omega_{f}\right)$, where $m_{f}$ and $\Omega_{f}$ are given by [14]

$$
\begin{gathered}
m_{f}=\frac{1}{\left(\frac{1}{m}-1\right) \frac{d(L, 2 \delta)}{d^{2}(L, \delta)}+1} \\
\Omega_{f}=\frac{d(L, \delta)}{N} \Omega_{0}
\end{gathered}
$$

respectively, where $d(L, \delta)=1-\mathrm{e}^{-L \delta} / 1-\mathrm{e}^{-\delta}$, while $\delta=$
0 , and then $m_{f}=L /(L-m+1)$, and $\Omega_{f}=L \Omega_{0} / N$. It is worth noting that the accuracy of the approximate expression becomes better when the number of multipaths, $L$, increase. After the channel fading model is modeled and established, we describe the receiver model, as shown in Figure 1, where the block diagram of the proposed $\mathrm{MC}$ technique is combined with the UWB system. There are $N$ subcarriers deployed in this infrastructure for the multiple access system. Each sub-channel signal is fed into a BPF followed by a notch filter demodulated by sinusoidal waveforms. The received signals are summed after dispreading and NRC combining with weight $g_{i}^{(k)}, i=0, \cdots, N-1$. The received signal $r(t)$ at the system output is expressed as

$$
\begin{aligned}
r(t) & n(t)+\sqrt{\frac{2 E_{b}}{N T_{b}}} \sum_{q=-\infty}^{\infty} \sum_{k=0}^{K-1} b^{(k)}(q) u_{T_{b}}\left(t-q T_{b}-\tau^{(k)}\right) \\
& \times \sum_{n=0}^{N-1} H_{n}^{(k)} c_{n}^{(k)} \cos \left(2 \pi f_{n} t+\theta_{n}^{(k)}\right)
\end{aligned}
$$

where $H_{n}^{(k)}=\beta_{n}^{(k)} \mathrm{e}^{j \theta_{n}^{(k)}}$ represents the sub-channel fading intensity with attenuation $\beta_{n}^{(k)}$ defined in (5), and $\theta_{n}^{(k)}$ is the phase of the nth subcarrier of the $k$ th user. The channel impulse response taps, $\left\{\alpha_{\ell}^{(k)}\right\}_{k=0}^{K-1}$, are assumed i.i.d (identically independent distributed) for different users with the same index $\ell$, thus,

$$
\left\{\alpha_{\ell}^{(k)}\right\}_{k=0}^{K-1} \sim M\left(\alpha_{\ell}^{(k)} ; m_{\ell}, \Omega_{\ell}\right),
$$

and

$$
\left\{\beta_{n}^{(k)}\right\}_{k=0}^{K-1} \sim M\left(\beta_{n}^{(k)} ; m_{f}, \Omega_{f}\right),
$$

$\tau^{(k)}$ is the time misalignment of user $k$ with respect to the referenced user at the receiver which is i.i.d for different users $k$ and uniformly distributed in $\left[q T_{b},(q+\right.$ 1) $T_{b}$ ]. $n(t)$ represents the AWGN (additive white Gaussian noise) having a two-side power spectral density of $N_{0} / 2$.

\section{Performance Analysis of Proposed System}

Without loss of generality, the 0 th user is considered the referenced user operating on each subcarrier suffering from a flat fading channel. Assuming that the channel fading, $\beta_{n}^{(k)}$, and phase shift, $\theta_{n}^{(k)}$, are constant over one bit duration. The decision variable, $U_{0, M C}$, of the 0th data bit of the referenced user after the coherent demodulation is given by

$$
U_{0, M C}=\frac{1}{T_{b}} \int_{0}^{T_{b}} r(t) \sum_{n=0}^{N-1} c_{n}^{(0)} g_{n}^{(0)} \cos \left(2 \pi f_{n} t\right) \mathrm{d} t
$$

where the weighting factors, $g_{n}^{(0)}$, of the 0 th user for an 


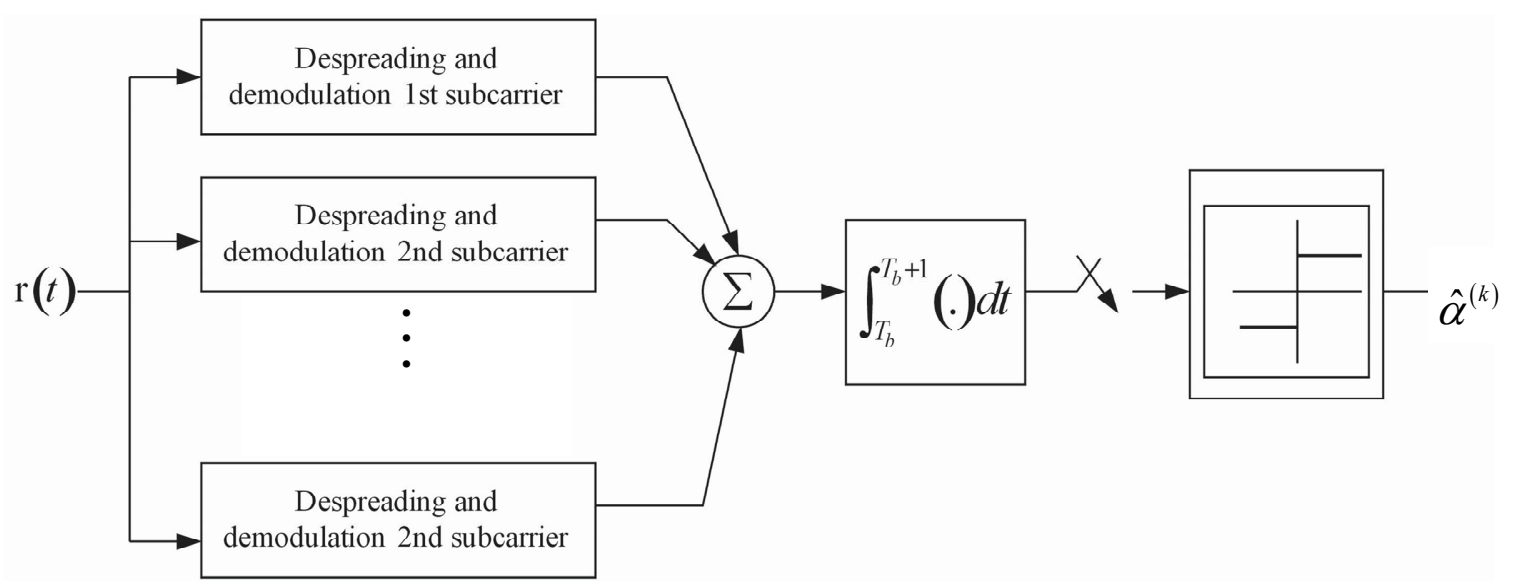

Figure 1. Block diagram of MC decision scheme combined with UWB system.

MRC (maximal ratio combining) diversity is employed in detection scheme, thus, let

$$
g_{n}^{(0)}=\left(H_{n}^{(0)}\right)^{*}=\beta_{n}^{(0)} \mathrm{e}^{-j \theta_{n}^{(0)}}
$$

By multiplying each subcarrier with the weighting factor $g_{n}^{(0)}$, and the decision variable, $U_{M C}^{(0)}$, after despreading the signal of the 0th user can be expressed as

$$
U_{M C}^{(0)}=D_{s}+J_{M A I}+J_{N}
$$

where the first term, $D_{s}$, denotes the desired signal of the referenced user can be calculated as (assuming that the case of $\left.b_{0}(0)=+1\right)$

$$
D_{s}=\sqrt{\frac{E_{b}}{2 N T_{b}}} \sum_{n=0}^{N-1}\left(\beta_{n}^{(0)}\right)^{2}
$$

the second term, $J_{M A I}$, in (11) indicates the MAI (multiple-access interference) which is including all the disturbed terms from the other located in the same BS (base station), and the last term, $J_{N}$, is the background noise component. Assuming that the number of multi-carriers $N$ is large enough, the chips for spreading code and the input data symbol are modeled as random variables, conditioned on channel state $\beta_{n}^{(0)}$. Hence, the MAI is able to be well approximated by zero mean Gaussian statistics and with variance obtained as [13]

$$
\sigma_{J_{M A I}}^{2}=\frac{E_{b}(K-1) \Omega_{f}}{4 N T_{b}} \sum_{n=0}^{N-1}\left(\beta_{n}^{(0)}\right)^{2}
$$

where $\Omega_{f}$ is given in (7). By utilizing the CLT (central limit theorem) definition, the random variable of $J_{N}$ can also be approximated using a zero mean Gaussian distribution with variance yields as

$$
\sigma_{J_{N}}^{2}=\frac{N_{0}}{4 T_{b}} \sum_{n=0}^{N-1}\left(\beta_{n}^{(0)}\right)^{2}
$$

Once the desired value and all statistical characteristic variances for the interference of the referenced user are computed, the instantaneous SINR (signal-to-interferencenoise ratio) can then be determined as

$$
\gamma_{M}=\left\{\left[\frac{2}{N} \sum_{n=0}^{N-1}\left(\beta_{n}^{(0)}\right)^{2}\right] /\left[(K-1) \frac{\Omega_{f}}{N}+\frac{N_{0}}{E_{b}}\right]\right\}
$$

where $E_{b} / N_{0}$ is the SNR per transmission bit. Now, the phenomena of the correlation characteristic between transmission branches should be taken into account. Different delay times could be caused for each path, when they arrive at the receiver via different propagation routes. Each path with the same delay may meet correlated fading among the separated paths. All of the possibilities may occur in the path of each subcarrier or the multi-path channel. In this article, the correlation will be ignored for the multi-path channel, since the spatial technique is not considered, thus, $h_{\ell}, \ell=0, \cdots, L-1$, are mutually uncorrelated for different $\ell$. It is believed that the correlation between the propagation channel cannot be canceled while the MC scheme is applied. However, the CFO (carrier frequency offset) is negligible when the channel estimation is considered. Hence, the correlation of $H_{n}$ and $H_{n^{\prime}}$ is expressed as

$$
\rho_{n n^{\prime}}=E\left[H_{n} H_{n^{\prime}}^{*}\right]=\frac{\Omega_{0}}{N} \frac{1-\mathrm{e}^{-\delta L / N} \mathrm{e}^{j 2 \pi\left(n^{\prime}-n\right) L / N}}{1-\mathrm{e}^{-\delta / N} \mathrm{e}^{j 2 \pi\left(n^{\prime}-n / N\right)}}
$$

It can be shown that in the case of $\delta=0$, in order to satisfy the case avoiding correlation, $\rho_{n n^{\prime}}=0$, the relationship between paths of

$$
n-n^{\prime}=c N / L
$$

must be satisfied, where nonzero integer $1 \leq c \leq L-1$. Among all sub-carriers, for a fixed sub-carrier $\mathrm{n}$, only $L-1$ other subcarriers are uncorrelated to sub-carrier $\mathrm{n}$. It is well known that when a random variable $x=\sqrt{\mu}$ is modeled with Nakagami- $m$ distribution, then the square of $x^{2}=\mu$, is a random variable characterized as Gamma distribution. Thus, the pdf of SINR, $\gamma_{M}$, shown in (15) can be denoted as 


$$
g(\gamma ; m, \Omega)=\left(\frac{m}{\Omega}\right)^{m} \frac{\gamma^{m-1}}{\Gamma(m)} \exp \left(-\frac{m}{\Omega} \gamma\right)
$$

where $\Omega$ and $m$ have been described in (4). By using of the symbol of $S_{M}^{C}$ express the summation of all the fading components at the system output, i.e., $S_{M}^{C}=\sum_{n=0}^{N-1}\left(\beta_{n}^{(0)}\right)^{2} / N$, since $\left\{\alpha_{\ell}^{(0)}\right\}_{\ell=0}^{L-1}$ are mutually uncorrelated, the statistical results of $S_{M}^{C}$ can be shown as $S_{M}^{C} \sim g\left(S_{M}^{C} ; m_{M}^{C}, \Omega_{M}^{C}\right)$, where

$$
m_{M}^{C}=\frac{E^{2}\left[S_{M}^{C}\right]}{\operatorname{var}\left[S_{M}^{C}\right]}=\frac{\left(\sum_{\ell=0}^{L-1} \mathrm{e}^{-\delta \ell}\right)^{2}}{\sum_{\ell=0}^{L-1} \frac{\mathrm{e}^{-2 \delta \ell}}{m}}=\frac{d^{2}(L, \delta)}{d(L, 2 \delta)} m
$$

and

$$
\Omega_{M}^{C}=\frac{d(L, \delta)}{N} \Omega_{0}
$$

where the first path arrival average power at the receiver output, and $d(L, \delta)$ are the same as shown in (7). By following the average system BER procedure for a coherent BPSK signaling system, the BER evaluation, $P_{U W B-M C-C D M A}$, for UWB system adoption in a MCCDMA system equipped with a MRC receiver can be calculated using the formula shown in [15]

$$
P_{U W B-M C-C D M A}=\int_{0}^{\infty} Q\left(\sqrt{\gamma_{M}}\right) g\left(x ; m, \Omega_{f}\right) \mathrm{d} x
$$

where $Q(\cdot)$ is the well known Gaussian $Q$-function, alternatively expressed as

$$
Q(x)=\frac{1}{\pi} \int_{0}^{\pi / 2} \exp \left(-\frac{x^{2}}{2 \sin ^{2} \theta}\right) \mathrm{d} \theta
$$

and by substituting (15) and (18) into (21), the average system BER result, $P_{U W B-M C-C D M A}$, of MC-CDMA system with MRC diversity scheme combined with UWB signaling can be obtained as (see the Appendix )

$$
\begin{aligned}
& P_{U W B-M C-C D M A}=\frac{1}{\pi} \int_{0}^{\frac{\pi}{2}}\left(\frac{m}{\Omega_{f}}\right)^{m} \\
& \left(\frac{\sin ^{-2} \theta}{\frac{(K-1) d(l, \delta)}{N^{2}} \Omega_{0}+\frac{N_{0}}{E_{b}}} \frac{T_{i}}{T_{i}+T_{g}}+\frac{m}{\Omega_{f}}\right)^{-m} \mathrm{~d} \theta
\end{aligned}
$$

where $d(l, \delta)=\frac{1-\exp (-l \delta)}{1-\exp (-\delta)}, \Omega_{f}=\frac{1}{N} d(l, \delta) \Omega_{0}$, with conditions of $0<\delta<1$ and $\Omega_{0}=1$.

\section{Numerical Results and Discussion}

In this section the UWB system performance combined with a MC-CDMA system is illustrated by implementation with a computer software package. Some of the parameters will be taken into account for accuracy validation and performance comparison, e.g., the number of received resolvable multi-paths, $L$, the user number, $k$, the fading parameter, $m$, and the sub-carrier number, $N$, of the MC-CDMA system. The role of correlation, which is always fixed at $\rho_{n n^{\prime}}=0.5$, is implicitly applied all through the numerical operation for evaluating the system performance. The bit duration is considered equal to that of a frame time, i.e., $T_{b}=T_{i}+T_{\mathrm{g}}$. First the BER versus SNR, $E_{b} / N_{0},(\mathrm{~dB})$ of the transmitted bits are illustrated in Figure 2. The user number is set as $K=2$, the received path number is $L=5$, and the sub-carrier number is set as $N=512$. In Figure 2 where different fading parameters, $m=1,3$ and 5 are considered, we adopted different values for $\delta=0.1,0.3$ and 0.5 . It is clear that the system performance was degraded by the fading parameter. However, the system performance will be independent of the power decay ratio parameter, $\delta$, after the SNR value increment is equal to $50 \mathrm{~dB}$. This fact tells us that the power decay ratio will disappear when the transmission energy symbol is increased. The system performance results with BER versus bit SNR are presented in Figure 3, with the effect of different numbers of users, $K=1,3$, and 4 . It is reasonable to note that the smaller number of users, the better the system performance. The system performance degradation is generated by the interference, which is caused by the number of different users. The effect comes from the resolvable multipath, $L$, provides the degradation of the system performance are presented in Figure 4. In advance, in Figure 4 where the received path number are assigned as 1 , 2 , and 5. Generally speaking it is well known that the little number of the received paths will deteriorate the system performance. As usual, the greater the fading

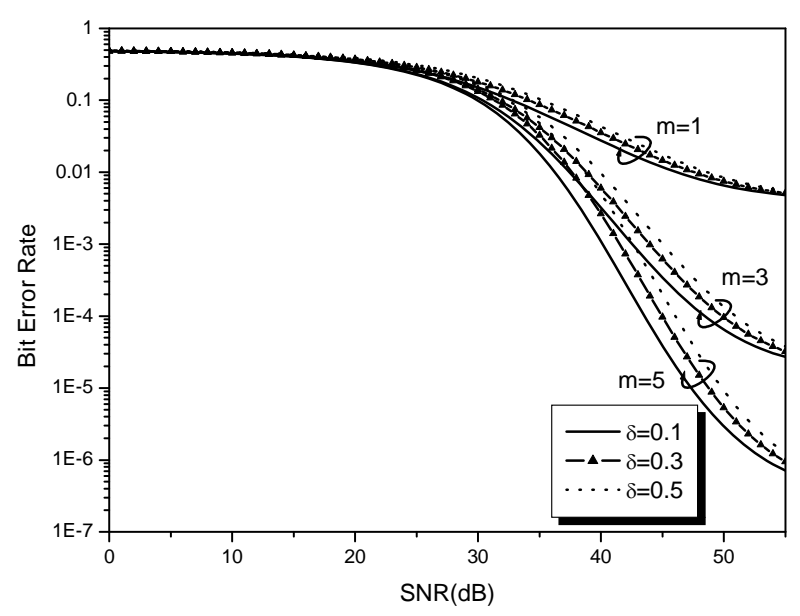

Figure 2. Plots of BER vs SNR (dB) with different parameters $\delta(k=2, L=5, N=512)$. 


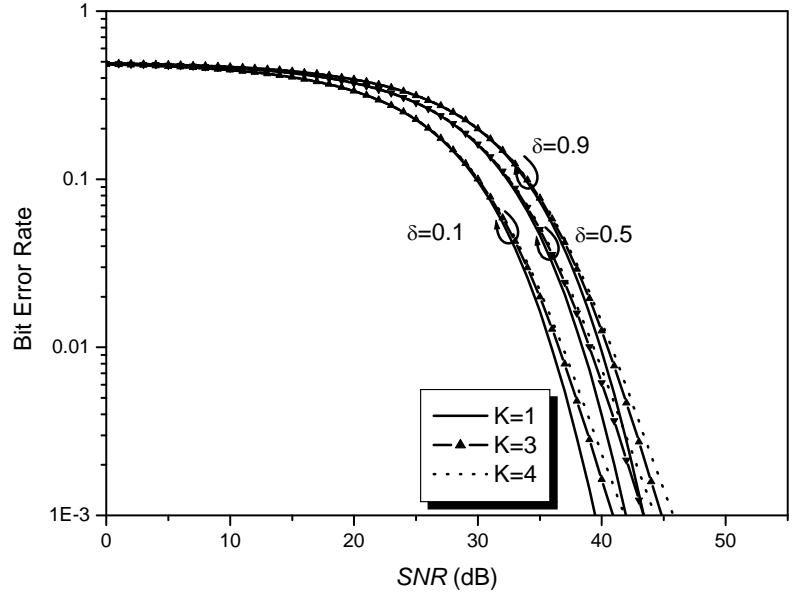

Figure 3. Plots of BER vs Eb/N0 (dB) with different parameters $K(m=5, L=5, N=512)$.

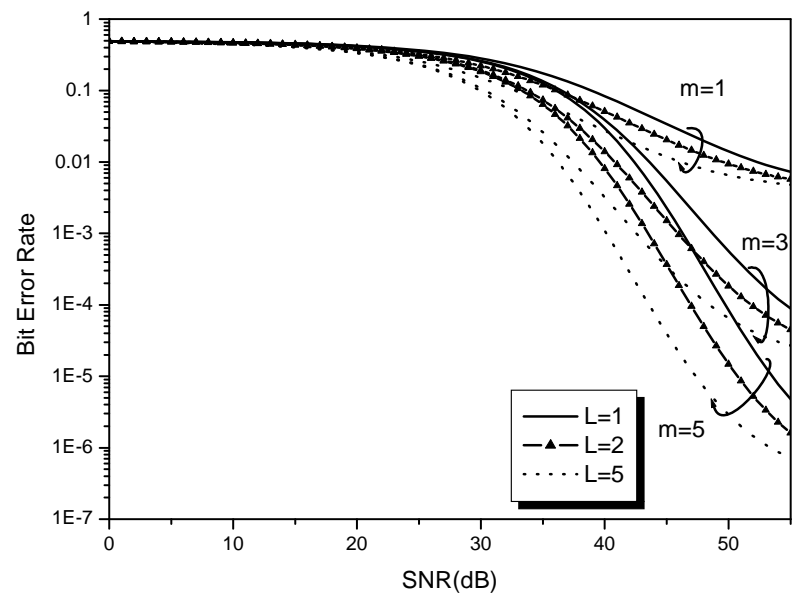

Figure 4. Plots of BER vs SNR (dB) with different parameters $L(k=2, \delta=0.1, N=125)$.

parameter value, the better the system performance. This fact can be explained as the system performance is dominated by the fading parameter. On the other hand, the system performance of an UWB system combined with $\mathrm{MC}$ techniques is dominated mainly by the fading environment. In contrast to the results shown in Figure 2, shows the system performance BER of the UWB system combined with a MC-CDMA system presented by the curves versus the $E_{b} / N_{0}(\mathrm{~dB})$ with different numbers of subcarriers, $N$, and different power decay ratios, $\delta$.

\section{Conclusions}

We proposed a system which adopted the UWB system in the MC system, i.e., the MC-CDMA system, in this paper. The system performance of the proposed configuration is also evaluated in the article. The fading channel is characterized as the correlated-Nakagami- $m$ distribu- tion. Some of the usual parameters were taken into account in the numerical analysis for accuracy validation and comparison. The most important thing worth noting is the fading parameter always dominates the system performance when considering the Nakagami- $m$ distribution as the fading model.

\section{REFERENCES}

[1] M. Schmidt and F. Jondral, "Ultra Wideband Transmission Based on MC-CDMA," IEEE Global Telecommunication Conference (Globecom'03,) San Francisco, 1-5 December 2003, pp. 749-753.

[2] K. Siwiak, "Ultra-Wide Band Radio: Introducing a New Technology," IEEE Vehicular Technology Conference, Rhodes, 6-9 May 2001, pp. 1088-1093.

[3] J. Foerster, "The Performance of a Direct-Sequence Spread Ultra-Wide Band System in the Presence of Multipath, Narrowband Interference, and Multiuser Interference," IEEE Ultra Wideband Systems and Technologies, Baltimore, 21-23 May 2002, pp. 87-91.

[4] J. Wang and L. B. Milstein, "Multicarrier CDMA Overlay for Ultra-Wideband Communications," IEEE Transactions on Communication, Vol. 52, No. 10, 2004, pp. 1664-1669. doi:10.1109/TCOMM.2004.836448

[5] M. Z. Win and R. A. Scholtz, "Ultra-Wide Bandwidth Time-Hopping Spread-Spectrum Impulse Radio for Wireless Multiple-Access Communications," IEEE Transactions on Communication, Vol. 48, No. 4, 2000.

[6] M. A. Rahman, S. Sasaki, S. T. Islam, T. Baykas, C. S. Sum, J. Wang, R. Funada, H. Harada and S. Kato, "Analysis and Comparison of Inter-Symbol/Frame Interference in Pulsed DS- and Hybrid DS/TH-UWB Communications," IEEE 69th Vehicular Technology Conference, Barcelona, 26-29 April 2009, pp. 1-5.

[7] A. F. Molisch, "Ultrawideband Propagation ChannelsTheory, Measurement, and Modeling," IEEE Transactions on Vehicular Technology, Vol. 54, No. 5, 2005, pp. 1528-1545. doi:10.1109/TVT.2005.856194

[8] A. Parihar, L. Lampe, R. Schober and C. Leung, "Equalization for DS-UWB Systems. Part I: BPSK Modulation," IEEE Transactions on Communication, Vol. 55, No. 6, 2007, pp. 1164-1173. doi:10.1109/TCOMM.2007.898836

[9] J. I. Montojo and L. B. Milstein, "Effects of Imperfections on the Performance of OFDM-Based UWB Systems," IEEE 42nd Conference on Signals, Systems and Computers, Pacific Grove, 26-29 October 2008, pp. $1985-$ 1989.

[10] S. Zhu, K. K. Leung and A. G. Constantinides, "Impacts of Impulse-Based Ultra-Wideband Data Links on Cooperative Wireless ad hoc Networks," IET Communications, Vol. 3, No. 2, 2009, pp. 321-330. doi:10.1049/iet-com:20080058

[11] Z. Dou, L. X. Sun and Y. Han, "Application of MUD in the Multi-User Communication of UWB System Based on Parallel Combinatory Spread Spectrum," 2011 Cross Strait Quad-Regional Radio Science and Wireless Technology Conference, Harbin, 26-30 July 2011, pp. 1732- 
1735.

[12] H. Shao and N. C. Beaulieu, "Direct Sequence and TimeHopping Sequence Designs for Narrowband Interference Mitigation in Impulse Radio UWB Systems," IEEE Transactions on Communication, Vol. 59, No. 7, 2011, pp. 1957-1965. doi:10.1109/TCOMM.2011.060911.100581

[13] J. I.-Z. Chen and T. W. Hsieh, "Another View Point on the Performance Evaluation of an MC-DS-CDMA System," Journal of Communications, and Networks, Vol. 11, No. 3, 2009, pp. 240-247.

[14] M. Nakagami, "The m-Distribtion: A General Formula of
Intensity Distribution of Rapid Fading," In: W. G. Hoffman, Ed., Statistical Methods in Radio Wave Propagation, Pergamon, Oxford, 1960.

[15] Z. J. Kang and K. Yao, "Performance Comparison of MC-CDMA over Frequency-Selective Nakagami- $m$ and Rayleigh Fadin Channels," IEEE 60th Vehicular Technology Conference, Los Angeles, 26-29 September 2004, pp. 4228-4232.

[16] I. S. Grodshteyn and I. M. Ryzhik, "Table of Integrals, Series, and Products," 5th Edition, Academic Press, San Diego, 1994. 


\section{Appendix}

In this appendix the Formula (23) is derived in detail.

From the SINR description shown in (15), it is known that the distribution has Gamma statistics. After put the SINR into (18), the Gamma distribution expression can be obtained. By substituting the Gamma distribution formula into (21) the result can be calculated as

$$
P_{U W B-M C-C D M A}=\int_{0}^{\infty} Q\left(\sqrt{\frac{2 x}{\frac{(K-1) d(l, \delta)}{N^{2}} \Omega_{0}+\frac{N_{0}}{E_{b}}} \frac{T_{i}}{T_{i}+T_{g}}}\right) \cdot\left(\frac{m}{\Omega_{f}}\right)^{m} \frac{x^{m-1}}{\Gamma(m)} \exp \left(-\frac{m}{\Omega_{f}} x\right) \mathrm{d} x
$$

where the $Q$-function can be alternatively replaced with the formula shown in (22), and the integral expression now becomes

$$
\begin{aligned}
& P_{U W B-M C \text {-CDMA }} \\
& =\frac{1}{\pi} \int_{0}^{\frac{\pi}{2}} \int_{0}^{\infty} \exp \left(\frac{-x \sin ^{-2} \theta}{\frac{(K-1) d(l, \delta)}{N^{2}} \Omega_{0}+\frac{N_{0}}{E_{b}}} \frac{T_{i}}{T_{i}+T_{g}}\right) \cdot\left(\frac{m}{\Omega_{f}}\right)^{m} \frac{x^{m-1}}{\Gamma(m)} \exp \left(-\frac{m}{\Omega_{f}} x\right) \mathrm{d} x \mathrm{~d} \theta \\
& =\frac{1}{\pi} \int_{0}^{\frac{\pi}{2}}\left(\frac{m}{\Omega_{f}}\right)^{m} \frac{1}{\Gamma(m)} \times \int_{0}^{\infty}\left(\frac{-x \sin ^{-2} \theta}{\frac{(K-1) d(l, \delta)}{N^{2}} \Omega_{0}+\frac{N_{0}}{E_{b}}} \frac{T_{i}}{T_{i}+T_{g}}-\frac{m x}{\Omega_{f}}\right) \exp x^{m-1} \mathrm{~d} x \mathrm{~d} \theta
\end{aligned}
$$

By applying the integral equivalent of the formula shown as [16]

$$
\int_{0}^{\infty} x^{v-1} \mathrm{e}^{-\mu x} \mathrm{~d} x=\frac{1}{\mu^{v}} \Gamma(v)
$$

and using of the variable changed with $v=m$, the other variable is denoted as

$$
\mu=\frac{\sin ^{-2} \theta}{\frac{(K-1) d(l, \delta)}{N^{2}} \Omega_{0}+\frac{N_{0}}{E_{b}}} \frac{T_{i}}{T_{i}+T_{g}}+\frac{m}{\Omega_{f}}
$$

By following the results illustrated in the previous equation, the indefinite integral in (A.2) becomes

$$
\int_{0}^{\infty} x^{m-1} \exp \left(-x\left(\frac{\sin ^{-2} \theta}{\frac{(K-1) d(l, \delta)}{N^{2}} \Omega_{0}+\frac{N_{0}}{E_{b}}} \frac{T_{i}}{T_{i}+T_{g}}+\frac{m}{\Omega_{f}}\right) \mathrm{d} x=\left(\frac{\sin ^{-2} \theta}{\frac{(K-1) d(l, \delta)}{N^{2}} \Omega_{0}+\frac{N_{0}}{E_{b}}} \frac{T_{i}}{T_{i}+T_{g}}+\frac{m}{\Omega_{f}}\right)^{-m} \Gamma(m)\right.
$$

The formula shown in (23) can be obtained by substituting (A.5) back into (A.2) and yielded as

$$
\begin{aligned}
P_{U W B-M C-C D M A} & =\frac{1}{\pi} \int_{0}^{\frac{\pi}{2}}\left(\frac{m}{\Omega_{f}}\right)^{m} \frac{1}{\Gamma(m)}\left(\frac{\sin ^{-2} \theta}{\frac{(K-1) d(l, \delta)}{N^{2}} \Omega_{0}+\frac{N_{0}}{E_{b}}} \frac{T_{i}}{T_{i}+T_{g}}+\frac{m}{\Omega_{f}}\right)^{-m} \Gamma(m) \mathrm{d} x \mathrm{~d} \theta \\
& =\frac{1}{\pi} \int_{0}^{\frac{\pi}{2}}\left(\frac{m}{\Omega_{f}}\right)^{m}\left(\frac{\sin ^{-2} \theta}{\frac{(K-1) d(l, \delta)}{N^{2}} \Omega_{0}+\frac{N_{0}}{E_{b}}} \frac{T_{i}}{T_{i}+T_{g}}+\frac{m}{\Omega_{f}}\right)^{-m} \mathrm{~d} \theta
\end{aligned}
$$

